\title{
SOME DIFFERENTIATION PROPERTIES OF MARKOFF TRANSITION PROBABILITY FUNCTIONS ${ }^{1}$
}

\author{
DONALD G. AUSTIN
}

In this paper we develop some differentiation properties of the stationary, transition, probability functions of a denumerable Markoff chain by a purely analytic method. That is, we consider analytic properties of a matrix of real valued functions $p_{i j}(t)(i, j=1,2, \cdots)$ for $0<t, t_{1}, t_{2}<\infty$ satisfying the conditions

$$
\begin{aligned}
\text { I } p_{i j}(t) & \geqq 0, \\
\text { II } \sum_{i} p_{i j}(t) & =1, \\
\text { III } p_{i j}\left(t_{1}+t_{2}\right) & =\sum_{k} p_{i k}\left(t_{1}\right) p_{k j}\left(t_{2}\right), \\
\text { IV } \quad \lim _{t \rightarrow 0} p_{i j}(t) & =\left\{\begin{array}{l}
0 \text { if } i \neq j, \\
1 \text { if } i=j .
\end{array}\right.
\end{aligned}
$$

The probabilistic significance of I, II, and III is clear if we consider events corresponding to the positive integers and $p_{i j}(t)$ as the conditional probability of the occurrence of event $j$ at time $t$ after the occurrence of event $i$. The probabilistic and mathematical significance of IV has been summarized by Doob in [1]. It has been shown by Doob [2] that the functions $p_{i j}(t)$ possess right-hand derivatives possibly infinite at $t=0$ and by Kolmogoroff [3] that this derivative is finite for $i \neq j$. Doob has considered the case $\sum_{j} D p_{i j}(0)$ $=0$ and shown that with this condition $p_{i j}(t)(j=1,2, \cdots)$ possess a continuous first derivative satisfying the backward differential equation of Kolmogoroff

$$
K_{1}: D p_{i j}(t)=\sum_{k} D p_{i k}(0) p_{i j}(t) .
$$

However, this condition doesn't appear sufficient for the Kolmogoroff forward equation

$$
K_{2}: D p_{i j}(t)=\sum_{k} p_{i k}(t) D p_{k j}(0) .
$$

Presented to the Society, December 29, 1954; received by the editors December 1, 1954.

1 This research was supported by the United States Air Force under Contract Number AF 18 (600)-760, monitored by the Office of Scientific Research. 
We have been informed that recently Kolmogoroff and Juskiewicz have shown by example that in general the functions $D p_{i j}(t)$ do not have first derivatives and, even with the assumption

$$
D p_{i i}(0)>-\infty
$$

they do not necessarily possess second derivatives.

This paper deals with two purely analytical problems, the question of the existence of derivatives and the question of analytical relations between them. In Theorem 1 we use condition (1) to establish bounds on the difference quotient and in Theorem 2 we use (1) to establish that the $p_{i j}(t)$ have a first derivative every where. This result (announced, with a brief proof in [6]) together with the results mentioned earlier presents a rather complete solution to the existence question $^{2}$ although it is still a question ${ }^{3}$ whether (1) implies that $p_{j i}(t)(j=1,2, \cdots)$ possess derivatives. Under (1) we show that $\sum_{j} D p_{i j}(t)=0, t>0$ (so that Doob's condition constitutes a restriction on the matrix only at the point 0 ) and that the differential equation

$$
D p_{i j}\left(t_{1}+t_{2}\right)=\sum_{k} D p_{i k}\left(t_{1}\right) p_{k j}\left(t_{2}\right)
$$

holds for $t_{1}>0, t_{2} \geqq 0$.

The special case where

$$
D p_{i i}(0)>-M \quad(i=1,2, \cdots)
$$

has been discussed by several authors. Feller [4] has shown that in this case the $p_{i j}(t)$ are analytic and satisfy both Kolmogoroff differential equations. We show in Theorem 3 that with condition (3) the $p_{i j}(t)$ satisfy the generalized Kolmogoroff equation

$$
D p_{i j}^{(m+n)}\left(t_{1}+t_{2}\right)=\sum_{k} D p_{i k}^{(m)}\left(t_{1}\right) D p_{k j}^{(n)}\left(t_{2}\right), \quad t_{1}, t_{2} \geqq 0 .
$$

We shall adopt the following notation

$$
\begin{array}{rlrl}
\Delta_{i j}\left(t_{1}, t_{2}\right) & =\left(p_{i j}\left(t_{2}\right)-p_{i j}\left(t_{1}\right)\right) /\left(t_{2}-t_{1}\right), & \\
\lim _{h \rightarrow 0} \Delta_{i j}(0, h) & =\left\{\begin{array}{cc}
q_{i j}, & i \neq j, \\
-q_{i}, & i=j
\end{array}\right.
\end{array}
$$

\footnotetext{
${ }^{2}$ I wish to thank Professor K. L. Chung for suggesting this problem to me and for helpful consultation.

${ }^{3}$ Added in proof. The author has now answered this question in the affirmative in his contract report USAF 18 (600)-760; September 1955.
} 
where we define $p_{i j}(0)=\delta_{i j}$. Also we mention two real variable theorems to which we shall have occasion to refer:

THEOREM A. The derivates and the difference quotient of a continuous function have the same least upper and greatest lower bounds (cf. [5, p. 74]).

THEOREM B. If the lower derivate of a finite function $f(x)$ is finite on $a$ set $K$ then $f(x)$ has a derivative almost everywhere (a.e.) on $K$ (cf. $[6, p p .269-271])$.

We mention three properties of the difference quotient that follow readily from I-IV:

$$
\begin{aligned}
& \sum_{i} \Delta_{i j}(t, t+h)=0, \\
& t \geqq 0, h>0, \\
& \Delta_{i j}(0, h)\left\{\begin{array}{l}
\geqq 0, \\
\leqq 0,
\end{array}\right. \\
& i \neq j \text {, } \\
& i=j \text {, } \\
& \Delta_{i j}\left(t_{1}+t_{2}, t_{1}+t_{2}+h\right)=\sum_{k} \Delta_{i k}\left(t_{1}, t_{1}+h\right) p_{k j}\left(t_{2}\right) \\
& =\sum_{k} p_{i k}\left(t_{1}\right) \Delta_{k j}\left(t_{2}, t_{2}+h\right) \text {. }
\end{aligned}
$$

The following theorem contains a condition for a uniform bound on the difference quotient of $p_{i j}(t)$.

THEOREM 1. If $q_{i}<\infty$ then

$$
q_{i} \geqq \Delta_{i j}\left(t_{1}, t_{2}\right) \geqq \operatorname{Max}\left(-q_{i},-q_{j}\right) .
$$

If either $q_{i}$ or $q_{j}<\infty$ then $p_{i j}(t)$ has a derivative almost everywhere.

Let $M>q_{i}$, then by (6) and the existence of the derivative at 0 there exists a $\delta>0$ such that $0 \geqq \Delta_{i i}(0, h)>-M$ whenever $0<h<\delta$. It then follows from (5) that

$$
0 \leqq \sum_{j \neq i} \Delta_{i j}(0, h)<M ; \quad 0<h<\delta .
$$

Let $t$ be any point on $(0, \infty)$, then, using (7), we have

$$
\begin{aligned}
-M<\Delta_{i i}(0, h) & \leqq \sum_{k} \Delta_{i k}(0, h) p_{k j}(t) \leqq \sum_{k \neq i} \Delta_{i k}(0, h) p_{k j}(t) \\
& \leqq \sum_{k \neq i} \Delta_{i k}(0, h)<M .
\end{aligned}
$$

That is $\left|\Delta_{i j}(t, t+h)\right|$ is less than or equal to $M$ and hence less than or equal to $q_{i}$ for $0<h<\delta$.

This shows that the right-hand derivates of $p_{i j}(t)$ are bounded 
by $q_{i}\left(\left|D_{+}^{+} p_{i j}(t)\right| \leqq q_{i}\right)$ and hence by Theorem ${ }^{4} \mathrm{~A}$ the difference quotient is in absolute value less than or equal to $q_{i}$. That is $p_{i j}(t)$ is Lipschitzian with constant $q_{i}$ and therefore of bounded variation and so by Lebesgue's theorem possesses a derivative almost everywhere.

Suppose $q_{j}<\infty$; again using (6) and (7) we see that $\Delta_{i j}(t, t+h)$ $=\sum_{k} p_{i k}(t) \Delta_{k j}(0, h) \geqq \Delta_{j j}(0, h)$ for any $t \geqq 0$. It follows that $D_{+} p_{i j}(t)$ is greater than or equal to $-q_{j}$. Thus by Theorems $\mathrm{A}$ and $\mathrm{B}$ we see that $p_{i j}(t)$ has a derivative almost everywhere. Further, in view of Theorem A, we may conclude that $\Delta_{i j}\left(t_{1}, t_{2}\right) \geqq-q_{j}$. Combining this with the Lipschitzian condition above we obtain (8).

REMARK. It may be noted that the proof of the lemma would have been only slightly complicated if we had replaced the condition $q_{i}<\infty$ by $D^{+} p_{i i}(0)>-\infty$, in which case we obtain an alternative proof of Doob's result that the $q_{i}$ always exist.

We now establish our basic theorem.

Theorem 2. If $q_{i}<\infty$ then $D p_{i j}(t)$ exists for $0<t<\infty$, is continuous, and satisfies

$$
\sum_{i} D p_{i j}(t)=0
$$

and

$$
D p_{i j}\left(t_{1}+t_{2}\right)=\sum_{k} D p_{i k}\left(t_{1}\right) p_{k j}\left(t_{2}\right), \quad \quad t_{1}>0 .
$$

Let us restrict ourselves to a bounded interval $I$ : $[0 \leqq t \leqq \bar{t}]$. By Dini's theorem on the convergence of monotone sequences of continuous functions we can, for any $\epsilon>0$, pick a $J$ such that $\sum_{j=J}^{\infty} p_{i j}(t)$ $\left\langle\epsilon / q_{i}\right.$ for $t \in I$. By Theorem 1 and (6) we have $0 \geqq \Delta_{i i}(0, h) \geqq-q_{i}$ and hence using (6) and (7)

$$
\Delta_{i j}(t, t+h)=\sum_{k} \Delta_{i k}(0, h) p_{k j}(t) \geqq-q_{i} p_{i j}(t) \quad \text { for any } t \text { and } h .
$$

Combining these inequalities we find that

$$
\sum_{j=J}^{\infty} \Delta_{i j}(t, t+h) \geqq \sum_{j=J}^{\infty}-q_{i} p_{i j}(t)>-\epsilon, \quad t \in I,
$$

and clearly this estimate holds over any sub-series, in particular if for fixed $t$ and $h$ we denote a summation over all negative $\Delta_{i j}(t, t+h)$,

4 The continuity of the $p_{i j}(t)$ was established by Doob in [2]. It is known that the $p_{i j}(t)$ are equi-continuous uniformly on $(0, \infty)$ : to see this, note that for any $\epsilon>0$ we may pick $\delta>0$ so that $1-p_{i i}(h)<\epsilon / 2$ whenever $0<h<\delta$ hence $\left|p_{i j}(t+h)-p_{i j}(t)\right|$ $=\left|\sum_{k}\left[p_{i k}(h)-p_{i k}(0)\right] p_{k j}(t)\right| \leqq \sum_{k}\left|p_{i k}(h)-p_{i h}(0)\right|=\sum_{k \neq i t p_{i k}(h)+1-p_{i i}(h)}$ $=2\left(1-p_{i i}(h)\right)<\epsilon$ for any $t \geqq 0$ and $j=1,2, \cdots$. 
$j=J, J+1, \cdots$ with a prime then

$$
\sum_{j=J}^{\infty} \Delta_{i j}(t, t+h)>-\epsilon .
$$

Using IV we pick $\eta$ such that $p_{k j}(s)<\epsilon / q_{i} J, k \neq j$, and $p_{j j}(s)>1-\epsilon / q_{i}$ for $0 \leqq s \leqq \eta$. Now for fixed $t_{1}<\bar{t}, s<\eta$ and arbitrary $h>0$ we have, in view of Theorem 1 ,

$$
\begin{aligned}
& \Delta_{i j}\left(t_{1}+s, t_{1}+s+h\right) \\
& =\sum_{k=J}^{\infty} \Delta_{i k}\left(t_{1}, t_{1}+h\right) p_{k j}(s)+\Delta_{i j}\left(t_{1}, t_{1}+h\right) p_{j j}(s) \\
& \quad+\sum_{k=1, k \neq j}^{J-1} \Delta_{i k}\left(t_{1}, t_{1}+h\right) p_{k j}(s) \geqq-3 \epsilon+\Delta_{i j}\left(t_{1}, t_{1}+h\right) .
\end{aligned}
$$

The inequality (13) along with Theorem $\mathrm{A}$ shows that $D^{+} p_{i j}\left(t_{1}\right)$ $=D_{+} p_{i j}\left(t_{1}\right)$, that is $p_{i j}(t)$ has a right-hand derivative $D_{R} p_{i j}(t)$ at $t=t_{1}$ and further $D_{R} p_{i j}(t)$ has the continuity property that for any $\epsilon>0$ there exists an $\eta>0$ such that for $0<s<\eta$ we have

$$
D_{R} p_{i j}(t+s)>D_{R} p_{i j}(t)-\epsilon .
$$

We now consider the series $\sum_{j} D_{R} p_{i j}(t)$. From (11) we see that $\sum$ ' $D_{R} p_{i j}(t) \geqq-q_{i}$ (where again we sum over negative terms) and thus $\sum_{j} D_{R} p_{i j}(t)$ converges, or diverges to $+\infty$. But by (5) and (12) we see that $\sum_{j} D_{R} p_{i j}(t) \leqq 0$ and hence the series converges. Also, it follows from Fatou's lemma that

$$
\begin{aligned}
D_{R} p_{i j}\left(t_{1}+t_{2}\right) & =\lim _{h \rightarrow 0^{+}} \sum_{k} \Delta_{i k}\left(t_{1}, t_{1}+h\right) p_{k j}\left(t_{2}\right) \\
& \geqq \sum_{k} \lim _{h \rightarrow 0^{+}} \Delta_{i k}\left(t_{1}, t_{1}+h\right) p_{k j}\left(t_{2}\right)=\sum_{k} D_{R} p_{i k}\left(t_{1}\right) p_{k j}\left(t_{2}\right)
\end{aligned}
$$

and hence

$$
\begin{aligned}
\sum_{i} D_{R} p_{i j}\left(t_{1}+t_{2}\right) & \geqq \sum_{j} \sum_{k} D_{R} p_{i k}\left(t_{1}\right) p_{k j}\left(t_{2}\right) \\
& =\sum_{k} D_{R} p_{i k}\left(t_{1}\right)\left[\sum_{j} p_{k j}\left(t_{2}\right)\right]=\sum_{k} D_{R} p_{i k}\left(t_{1}\right)
\end{aligned}
$$

That is, we have now shown that $\sum_{j} D_{R} p_{i j}(t)$ is convergent, nonpositive series, monotone increasing in $t$. We now show that for any $\epsilon$ the set of points $t$ where $\sum_{j} D_{R} p_{i j}(t)>-\epsilon$ is dense and this enables us to conclude that

$$
\sum_{j} D_{R} p_{i j}(t) \equiv 0
$$


Let $\sum_{j} D_{R} p_{i j}(t)=-A$ and pick $J$ sufficiently large that (12) holds for the given $\epsilon$ and such that

$$
\begin{aligned}
& \text { (i) }\left|\sum_{j=1}^{J} D_{R} p_{i j}(t)+A\right|<\epsilon \text { and } \\
& \text { (ii) }\left|\sum_{j=1}^{J} \Delta_{i j}(t, t+h)+A\right|<\epsilon
\end{aligned}
$$

for $h$ less than some number $\delta$. By (14) we may take $\delta$ sufficiently small that for $h<\delta$ we have also

$$
\sum_{j=1}^{J} D_{R} p_{i j}(t+h)>\sum_{j=1}^{J} D_{R} p_{i j}(t)-\epsilon .
$$

Now let us fix $h_{1}<\delta$ and pick $J_{1}>J$ such that $\sum_{j=J+1}^{J_{1}} \Delta_{i j}\left(t, t+h_{1}\right)$ $>A-\epsilon$; this is possible by (5). But by Theorem A there exists an $h_{2}<h_{1}$ such that

$$
\sum_{j=J+1}^{J_{1}} D_{R} p_{i j}\left(t+h_{1}\right)>A-\epsilon .
$$

By our choice of $J$ we obtain, using (12), that

$$
\sum_{j=J_{1}+1}^{\infty} D_{R} p_{i j}\left(t+h_{1}\right)>-\epsilon .
$$

Finally combining (17), (18), (19), and (20) we see that $\sum_{j=1}^{\infty} D_{R} p_{i j}(t$ $\left.+h_{1}\right)>-4 \epsilon$ and this is our desired density result.

We have now strengthened (5) to hold for $h \geqq 0$ where by $h=0$ we mean the right-hand derivatives. With this fact and condition (12), Dini's argument on the uniform convergence of a monotone sequence of continuous functions with a continuous limit may be applied to the sequence $\sum_{j=1}^{J} \Delta_{i j}(t, t+h)$ as functions of $h$ on the closed set $h \geqq 0$ to conclude that this series converges uniformly in $h$.

We have now, in view of the Moore-Osgood theorem, justified an interchange of limits to strengthen (15)

$$
\begin{aligned}
D_{R} p_{i j}(t+s) & =\lim _{h \rightarrow 0} \sum_{k} \Delta_{i k}(t, t+h) p_{k j}(s) \\
& =\sum_{k} \lim _{h \rightarrow 0} \Delta_{i k}(t, t+h) p_{k j}(s)=\sum_{k} D_{R} p_{i k}(t) p_{k j}(s) .
\end{aligned}
$$

However, since the $p_{k j}(s)$ are continuous and $\sum_{k} D_{R} p_{i k}(t)$ convergent, we see that the right-hand side of (21) is a continuous function of $s$ and this implies the continuity of the right derivative of $p_{i j}(t)$ and hence by Theorem $A$ the existence and continuity of the derivative. 
Statements (ii) and (iii) of the theorem now follow immediately from (16) and (21). ${ }^{5}$

Let us now consider the differentiability problem under the more restrictive hypothesis that the $q_{i}(i=1,2, \cdots)$ are bounded. As mentioned in the introduction, this condition has been discussed by Feller [4] and Theorem 3 of this paper contains some of his results. We first prove a real variable lemma. Let $g_{k}(x)(k=1,2, \ldots)$ be a sequence of functions having continuous derivatives on an interval $I[x ; a \leqq x \leqq b]$ and let $\Delta_{f}(x, y)=(f(y)-f(x)) /(y-x)$ for any function $f(x)$.

Lemma 1. If $\sum_{k}\left|D g_{k}(x)\right|$ converges uniformly on I then $\sum_{k} \mid \Delta g_{k}(x$, $x+h) \mid$ converges uniformly in $(x, h)$ for $h>0, x$ and $x+h$ in $I$.

For given $\epsilon>0$ we may choose $N$ so that for $n>N$ we have $\sum_{k=n}^{\infty}\left|D g_{k}(x)\right|<\epsilon$. Now

$$
\begin{aligned}
\sum_{k=n}^{\infty}\left|\Delta g_{k}(x, x+h)\right| & =\sum_{k=n}^{\infty} \frac{1}{h} \int_{x}^{x+h} D g_{k}(x) d x \\
& \leqq \frac{1}{h} \int_{x}^{x+h} \sum_{k=n}^{\infty}\left|D g_{k}(x)\right| d x \leqq \epsilon
\end{aligned}
$$

providing that $x$ and $x+h$ are in $I$, and this completes the proof. ${ }^{6}$

The following lemma indicates that if the $q_{i}$ are bounded then the $p_{i j}(t)$ have derivatives of all orders which have a bound depending only on the order of the derivative, that is independent of $i, j$, and $t$. We adopt the following notation: $D^{(n)} p_{i j}(t)$ is the $n$th derivative of $p_{i j}(t)$ at $t ; D^{(0)} p_{i j}(t)=p_{i j}(t)$; and

$$
\Delta_{i, j}^{(n)}\left(t_{1}, t_{2}\right)=\frac{D^{(n-1)} p_{i j}\left(t_{2}\right)-D^{(n-1)} p_{i j}\left(t_{1}\right)}{t_{2}-t_{1}} .
$$

Lemma 2. If $q_{i}<M(i=1,2, \cdots)$ then the $p_{i j}(t)$ have derivatives of all orders on $(0, \infty)$, with $\left|D^{(n)} p_{i j}(t)\right| \leqq(2 M)^{n}$, which satisfy the differential equation:

$$
D^{(n)} p_{i j}\left(t_{1}+t_{2}\right)=\sum_{k} p_{i k}\left(t_{1}\right) D^{(n)} p_{k j}\left(t_{2}\right) .
$$

We prove the theorem by induction. By (I) and (III) the theorem is true for $n=0$. Suppose that the theorem is true for $n=\alpha-1$, that

${ }^{5}$ The methods of this proof may be used to strengthen the last statement of Theorem 1 to read that if $q_{i}<\infty$ then $p_{i j}(t)$ has a right-hand derivative at all points, but this result seems rather incomplete.

' This short proof of the lemma was suggested by 'the referee.'. 
is, suppose $p_{i j}(t)$ has derivatives up to order $\alpha-1$ with $\left|D^{(n)} p_{i j}(t)\right|$ $\leqq(2 M)^{n}$ for $n=0,1, \cdots, \alpha-1$ satisfying (22). By (22), with $n=\alpha-1$, we see that for arbitrary $t, h>0$;

$$
\Delta_{i j}^{(\alpha)}(t, t+h)=\sum_{k} \Delta_{i k}(0, h) D^{(\alpha-1)} p_{k j}(t) .
$$

Given an $\epsilon>0$, let $\delta<0$ be such that for $0<h<\delta$ we have $\Delta_{i i}(0, h)$ $>-q_{i}-\epsilon>-M$ and $\sum_{j \neq i} \Delta_{i j}(0, h)<q_{i}+\epsilon<M$. By (23) we see that

$$
\begin{aligned}
\left|\Delta_{i j}^{(\alpha)}(t, t+h)\right| & \leqq \sum_{k}\left|\Delta_{i k}(0, h)\right|\left|D^{(\alpha-1)} p_{k j}(t)\right| \\
& \leqq(2 M)^{\alpha-1} \sum_{k}\left|\Delta_{i k}(0, h)\right| \\
& =(2 M)^{\alpha-1}\left\{\sum_{k \neq i} \Delta_{i k}(0, h)-\Delta_{k k}(0, h)\right\} \leqq(2 M)^{\alpha} .
\end{aligned}
$$

From this we may conclude, exactly as in the proof of Theorem 1, that $D^{(\alpha-1)} p_{i j}(t)$ is Lipschitzian with constant $(2 M)^{\alpha}$.

Now let $t$ be any point and let $\bar{t}<t$ be some point where each of the functions $p_{k j}(t)(k=1,2, \cdots)$ has a derivative of order $\alpha$; such a point exists by the last paragraph in view of Lebesgue's theorem. Now consider:

$$
\Delta_{i j}^{(\alpha)}(t, t+h)=\sum_{k} p_{i k}(t-\bar{t}) \Delta_{k j}^{(\alpha)}(\bar{t}, \bar{t}+h)
$$

which is valid by the induction hypothesis. We know (i) $\sum_{k} p_{i k}(t-\bar{t})$ converges, (ii) $\lim _{h \rightarrow 0} \Delta_{i j}^{(\alpha)}(\bar{t}, \bar{t}+h)$ exists, and (iii) $\left|\Delta_{i j}^{(\alpha)}(\bar{t}, \bar{t}+h)\right|$ $\leqq(2 M)^{\alpha}$, hence we may use the Weierstrass $M$-test and then the Moore-Osgood theorem to conclude that $\lim _{h \rightarrow 0} \Delta_{i, j}^{(\alpha)}(t, t+h)$ exists and

$$
D^{(\alpha)} p_{i j}(t)=\sum_{k} p_{i k}(t-\bar{t}) D^{(\alpha)} p_{k j}(\bar{t}) .
$$

Thus we see that each of the $p_{i j}(t)$ possess derivatives of order $\alpha$ at all points of $(0, \infty)$. But the only property of $\bar{t}$ used above was that each of the functions $p_{k j}(t)(k=1,2, \cdots)$ had derivatives of order $\alpha$ at $t=\bar{t}$ and so the completion of the induction (that is, the verification of (25) for arbitrary $\bar{t}$ and $(t-\bar{t})$ ) is immediate.

The following lemma extends the differentiability conclusions of Lemma 2 to include $t=0$ and contains a convergence criteria for the derivatives of $p_{i j}(t), j=1,2, \cdots$.

LeMma 3. If $q_{i}<M(i=1,2, \cdots)$ then: 
(1) The $p_{i j}(t)$ have continuous derivatives of all orders on $[0, \infty)$.

(2) The series $\sum_{j}\left|D^{(n)} p_{i j}(t)\right|$ converges uniformly on any finite closed interval I contained in the non-negative real axis and $\sum_{j} D^{(n)} p_{i j}(t)$ $\equiv 0$.

(3) The differential equation:

$$
D^{(n)} p_{i j}\left(t_{1}+t_{2}\right)=\sum_{k} D^{(n)} p_{i k}\left(t_{1}\right) p_{k j}\left(t_{2}\right)
$$

is satisfied for $t_{1}, t_{2} \geqq 0$.

The theorem is clearly true for $n=0$.

Suppose it is true for $n=\alpha-1$; we then have

$$
\Delta_{i j}^{(\alpha)}\left(t_{1}+t_{2}, t_{1}+t_{2}+h\right)=\sum_{k} D^{(\alpha-1)} p_{i k}\left(t_{1}\right) \Delta_{k j}\left(t_{2}, t_{2}+h\right),
$$

where $t_{2} \geqq 0, h>0$, and $t_{1} \in I$. Now, in view of the induction hypothesis, Lemma 2, and the existence of $q_{i}$ and $q_{i j}$, we may apply the Moore-Osgood theorem to take the limit as $h \rightarrow 0$ inside the summation sign to obtain:

$$
D^{(\alpha)} p_{i j}\left(t_{1}+t_{2}\right)=\sum_{k} D^{(\alpha-1)} p_{i k}\left(t_{1}\right) D^{(1)} p_{k j}\left(t_{2}\right) .
$$

Note that the existence of $D^{(\alpha)} p_{i j}(0)$ follows from the Moore-Osgood theorem on placing $t_{1}=t_{2}=0$; the bound on the derivative is immediate. From (28) with $t_{2}=0$ we have, for any integer $J>0$ :

$$
\begin{aligned}
\sum_{j=J}^{\infty}\left|D^{(\alpha)} p_{i j}(t)\right| & \leqq \sum_{j=J}^{\infty}\left|\sum_{k \neq j} D^{(\alpha-1)} p_{i k}(t) q_{k j}\right|+\sum_{j=J}^{\infty}\left|D_{i j}^{(\alpha-1)}(t)\right| q_{j} \\
& \leqq \sum_{j=J}^{\infty} \sum_{k \neq j}\left|D^{(\alpha-1)} p_{i k}(t)\right| q_{k j}+\sum_{j=J}^{\infty}\left|D^{(\alpha-1)} p_{i j}(t)\right| q_{j} \\
& =\sum_{k} \sum_{j=J, j \neq k}^{\infty}\left|D^{(\alpha-1)} p_{i k}(t)\right| q_{k j}+\sum_{j=J}^{\infty}\left|D^{(\alpha-1)} p_{i j}(t)\right| q_{j},
\end{aligned}
$$

where the change in order of summation follows from the MooreOsgood theorem in view of the induction hypothesis and the bound on $q_{k j}$ (i.e. $\left.q_{k j} \leqq M\right)$. For any $\epsilon>0$ we may pick $K$ so that $\sum_{k=K}^{\infty}\left|D^{(\alpha-1)} p_{i k}(t)\right|<\epsilon / M$, for $t \in I$, by the induction hypothesis; we then pick $J>K$ so that

$$
\sum_{j=J}^{\infty} q_{k i}<\epsilon /(K-1)(2 M)^{\alpha-1} \text { for } k=1,2, \cdots, K-1 .
$$

Substituting into (29) we find that 


$$
\begin{aligned}
& \sum_{j=J}^{\infty}\left|D^{(\alpha)} p_{i j}(t)\right| \\
& \quad \leqq \sum_{k=1}^{K-1} \sum_{j=J, j \neq k}^{\infty}\left|D^{(\alpha-1)} p_{i k}(t)\right| q_{k j}+\sum_{k=K}^{\infty} \sum_{j=J, j \neq k}^{\infty}\left|D^{(\alpha-1)} p_{i k}(t)\right| q_{k j} \\
& \quad+\sum_{j=J}^{\infty}\left|D^{(\alpha-1)} p_{i j}(t)\right| q_{j} \leqq(2 M)^{\alpha-1}(K-1) \frac{\epsilon}{(K-1)(2 M)^{\alpha-1}} \\
& \quad+\frac{\epsilon}{M} M+\frac{\epsilon}{M} M=3 \epsilon
\end{aligned}
$$

for $t \in I$. This implies that $\sum_{j}\left|D^{(\alpha)} p_{i j}(t)\right|$ converges uniformly on $I$. This fact, with $\sum_{j} \Delta_{i j}^{(\alpha)}(t, t+h) \equiv 0$ for $h>0$ implied by the induction hypothesis, shows that $\sum_{j} D^{(\alpha)} p_{i j}(t) \equiv 0$.

Now let $t_{1}$ be any point in $I, h \geqq 0$ any number so that $t_{1}+h \in I$; then again using the induction hypothesis:

$$
\Delta_{i j}^{(\alpha)}\left(t_{1}+t_{2}, t_{1}+t_{2}+h\right)=\sum_{k} \Delta_{i k}^{(\alpha)}\left(t_{1}, t_{1}+h\right) p_{k j}\left(t_{2}\right)
$$

for any $t_{2} \geqq 0$. But by Lemma 1 the series in (30) converges uniformly in $h$, hence we may take the limit as $h \rightarrow 0$ under the summation sign to obtain (26) for $n=\alpha$. Since $I$ was any finite interval with lefthand end point 0 , the number $t_{1}$ is arbitrary and the induction is complete. $^{7}$

We now summarize and extend Lemmas 2 and 3.

Theorem 3. If $q_{i}<M$ then all of the functions $p_{i j}(t)$ have derivatives of all orders on $[0, \infty)$ satisfying the differential equation:

$$
D^{(m+n)} p_{i j}\left(t_{1}+t_{2}\right)=\sum_{k} D^{(m)} p_{i k}\left(t_{1}\right) D^{(n)} p_{k j}\left(t_{2}\right)
$$

for $t_{1}, t_{2}$ in the closed interval $[0, \infty)$ and $m, n=0,1,2, \cdots$. Further $p_{i j}(t)$ may be expanded in a Taylor series convergent on $[0, \infty)$.

The first statement is included in Lemma 3, (1). We prove the second statement by induction. It is trivially true for $m+n=0$. Suppose that it is true for $m+n=\alpha-1$ and let $\bar{m}$ and $\tilde{n}$ be any two nonnegative integers such that $\bar{m}+\bar{n}=\alpha$. By Lemma 3, (3) formula (31) holds if $\bar{n}=0$ so we may consider $\bar{n}>0$. By the induction hypothesis we have

7 In addition to Feller's paper [4] this case has been considered by Arley [5]. Arley has obtained the differential equation (26) with a somewhat weaker restriction than $q_{i}$ bounded. 


$$
\Delta_{i, j}^{(\alpha)}\left(t_{1}+t_{2}, t_{1}+t_{2}+h\right)=\sum_{k} D^{(\bar{m})} p_{i k}\left(t_{1}\right) \Delta_{k j}^{(\tilde{n})}\left(t_{2}, t_{2}+h\right),
$$

for $t_{1}, t_{2} \geqq 0, h>0$. Now, in view of Lemmas 2 and 3 we may apply the Moore-Osgood theorem to obtain (31).

The Taylor expansion follows from Lemma 3, (1) and the result that a Taylor expansion converges to the function on $[0, \infty)$ if on every finite closed interval with left end point 0 the function has derivatives of all orders (right-hand derivatives at 0$), D^{(n)} p_{i j}(t)$, such that the Cauchy remainder $\left(h^{n} /(n-1) !\right) D^{(n)} p_{i j}(\theta h)(1-\theta)^{n-1}$ converges to 0 as $n$ tends to infinity, for fixed $h$, uniformly for $\theta \in[0,1]$ $\{$ cf. $[7$, p. 209]\}.

\section{BIBLIOGRAPHY}

1. J. L. Doob, Markoff chains-denumerable case, Trans. Amer. Math. Soc. vol. 58 (1945) pp. 455-472.

2. - Topics in the theory of Markoff chains, Trans. Amer. Math. Soc. vol. 52 (1942) pp. 37-64.

3. A. N. Kolmogoroff, Ucenye Zapiski (Matem.), Moskov. Gor. Univ., vol. 148, pp. 53-59.

4. W. Feller, On the integro-differential equations of purely discontinuous Markoff processes, Trans. Amer. Math. Soc. vol. 48 (1940) pp. 488-515.

5. M. Arley, Stochastic processes and cosmic radiation, Copenhagen, 1943.

6. D. G. Austin, On the existence of the derivative of Markoff transition probability functions, Proc. Nat. Acad. Sci. vol. 41 (1941) pp. 224-226.

7. E. W. Hobson, Theory of functions of a real variable, vol. II, Harren Press, 2d ed.

Syracuse University 